\title{
A Characterisation of Morita Algebras in Terms of Covers
}

\section{Tiago Cruz ${ }^{1}$ (D)}

Received: 27 January 2021 / Accepted: 2 May 2021 / Published online: 4 June 2021

(C) The Author(s) 2021

\begin{abstract}
A pair $(A, P)$ is called a cover of $\operatorname{End}_{A}(P)^{o p}$ if the $\operatorname{Schur}$ functor $\operatorname{Hom}_{A}(P,-)$ is fully faithful on the full subcategory of projective $A$-modules, for a given projective $A$-module $P$. By definition, Morita algebras are the covers of self-injective algebras and then $P$ is a faithful projective-injective module. Conversely, we show that $A$ is a Morita algebra and $\operatorname{End}_{A}(P)^{o p}$ is self-injective whenever $(A, P)$ is a cover of $\operatorname{End}_{A}(P)^{o p}$ for a faithful projective-injective module $P$.
\end{abstract}

Keywords Morita algebras · Covers · Self-injective algebras

Mathematics Subject Classification (2010) $16 \mathrm{G} 10 \cdot 16 \mathrm{~S} 50 \cdot 16 \mathrm{~L} 60$

\section{Introduction}

Morita algebras were introduced in [8] to better understand and generalize self-injective algebras. The definition is based on a theorem by Morita (see [9, section 16], [8, p. 185]) and it says that a Morita algebra is the endomorphism algebra of a generator over a self-injective algebra. Moreover, Morita showed that this generator can be chosen to be projectiveinjective of the form $A e \simeq D(e A)$ when regarded as a left module over the Morita algebra $A$, for some idempotent $e$ of $A$. Modules containing the regular module as a direct summand are examples of generators.

Morita algebras occur in several contexts, including cover theory and the MoritaTachikawa correspondence.

A cover, in Rouquier's sense [11], of an algebra $B$ is a pair $(A, P)$ consisting of the endomorphism algebra $A$ of a generator over $B$ and a certain projective $A$-module $P$. Covers

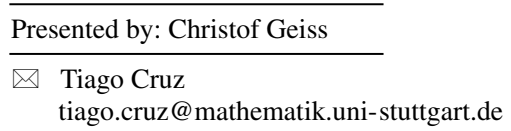

1 Institute of Algebra and Number Theory, University of Stuttgart, Pfaffenwaldring 57, 70569

Stuttgart, Germany 
are useful to transfer properties from the cover to $B$ through a $\operatorname{Schur}$ functor $\operatorname{Hom}_{A}(P,-)$. This construction allows us to view the module category of $B$ as a kind of quotient of the module category of its cover $A$. It follows from their definition that Morita algebras are exactly the covers of self-injective algebras.

On the other hand, generators over self-injective algebras are also cogenerators. The endomorphism algebras of generators-cogenerators are described by the Morita-Tachikawa correspondence, which classifies the finite-dimensional algebras with dominant dimension at least two as the endomorphism algebras of a generator-cogenerator. The famous Nakayama conjecture claims that finite-dimensional algebras with infinite dominant dimension are self-injective.

Many interesting covers arise as endomorphism algebras of generators-cogenerators. In this situation, the following questions arise. Given a faithful projective-injective $A$-module $P$ :

- When is $(A, P)$ a cover of $\operatorname{End}_{A}(P)^{o p}$ ?

- When is $A$ a Morita algebra?

- When is $\operatorname{End}_{A}(P)^{o p}$ a self-injective algebra?

Our main result provides answers to these questions and it provides several characterisations of Morita algebras with fewer assumptions than the theorem by Morita that motivated the definition of Morita algebras in [8, pages 185-186]:

Theorem 1 Let A be a finite-dimensional algebra. Assume that $P$ is a faithful projectiveinjective left A-module. Then the following assertions are equivalent:

(i) $(A, P)$ is a cover of $\operatorname{End}_{A}(P)^{o p}$;

(ii) A is a Morita algebra;

(iii) The endomorphism algebra $\operatorname{End}_{A}(P)^{o p}$ is a self-injective algebra and $\operatorname{domdim} A \geq 2$;

(iv) $\operatorname{domdim} A \geq 2$ and $\operatorname{add}_{A} D A \otimes_{A} P=\operatorname{add}_{A} P$.

(v) $\operatorname{dom} \operatorname{dim} A \geq 2$ and the Nakayama functor restricts to $D A \otimes_{A}-: \operatorname{add}_{A} P \rightarrow \operatorname{add}_{A} P$.

The implications $(\mathrm{ii}) \Leftrightarrow$ (iii) $\Longrightarrow($ i $)$ are already known by [9, section 16] and Morita-Tachikawa correspondence. The equivalence $(v) \Leftrightarrow(i v) \Leftrightarrow(i i)$ is related to the study of strongly projective modules in [4]. Here, we present a shorter proof. The proof of Theorem 1 involves the study of double centralizer properties and a reformulation of the definition of Morita algebras using the Nakayama functor. Prominent examples of double centralizer properties are Soergel's double centralizer theorem [12], classical Schur-Weyl duality [6] and its many generalizations (see for example [2]).

As a byproduct of Theorem 1, we clarify in Remark 13 some situations where a double centralizer property on a module $A e$ is equivalent to a double centralizer property on $e A$, for some idempotent $e$ of a given finite-dimensional algebra $A$. Further, although it does not come as a surprise, we see in Example 17 that if $P$ is only projective the assertion $(i)$ together with $\left(A, \operatorname{Hom}_{A}(P, A)\right)$ being a cover of $\operatorname{End}_{A}(P)^{o p}$ is not sufficient for $A$ to be a Morita algebra.

As application of Theorem 1, we give in Corollary 14 a criterion for a $Q F-1$ algebra to be a self-injective algebra. 


\section{Notation}

We will assume throughout this paper that $k$ is a field and $A$ and $B$ are finite-dimensional $k$-algebras. By $A$-mod (resp. mod- $A$ ) we mean the category of finitely generated left (resp. right) $A$-modules and by $A$-proj the full subcategory of $A$-mod whose modules are the finitely generated projective $A$-modules. We denote by (resp. add $M_{A}$ ) (or just add $M$ when $A$ is fixed) the full subcategory of $A$-mod (resp. mod- $A$ ) whose modules are direct summands of finite direct sums of $M \in A$-mod (resp. $M \in \bmod -A$ ). We write $A$-proj to denote $\operatorname{add} A$. For any $M \in A$-mod and $f, g \in \operatorname{End}_{A}(M)$ the multiplication $f g$ is the composite $f \circ g$ of $g$ and $f$. The opposite algebra of $A$ will be denoted by $A^{o p}$.

Given a finitely generated $(A, B)$-bimodule $M$, there is a double centralizer property on $M$ between $A$ and $B$ provided that the multiplication maps on $M$ induce isomorphisms $A \simeq \operatorname{End}_{B}(M)$ and $B \simeq \operatorname{End}_{A}(M)^{o p}$. By the standard duality $D$ we mean the functor $\operatorname{Hom}_{k}(-, k): A$-mod $\rightarrow A^{o p}$-mod.

An algebra $B$ is called self-injective if the regular module $B$ is an injective left $B$ module, or, equivalently, if the regular module $B$ is a right $B$-module. If there exists a ( $B, B$ )-bimodule isomorphism between $D B$ and $B$ then $B$ is called a symmetric algebra.

\section{Dominant Dimension}

Let

$$
0 \rightarrow{ }_{A} A \rightarrow I_{0} \rightarrow I_{1} \rightarrow \cdots \rightarrow I_{n} \rightarrow \cdots
$$

be a minimal injective resolution of the regular module ${ }_{A} A$. We say that the dominant dimension of the algebra $A$, denoted by $\operatorname{dom} \operatorname{dim} A$, is $n \in \mathbb{N} \cup\{\infty\}$ if $I^{t}$ is projective for $t<n$ and $I_{n}$ is not. In particular, $\operatorname{dom} \operatorname{dim} A$ is infinite if all injective modules $I_{t}$ are projective. Analogously, we can define the dominant dimension using the right regular module $A_{A}$. This (right) dominant dimension is equal to $\operatorname{dom} \operatorname{dim} A$. A detailed account on dominant dimension can be found in $[10,13]$. $A$ is called $Q F-3$ algebra if $\operatorname{domdim} A \geq 1$. In such a case, $I_{0}$ is a faithful projective-injective module. Moreover, given another faithful projective-injective module $X \in A-\bmod , \operatorname{add} X=\operatorname{add} I_{0}[7, \operatorname{Lemma} 2.3]$ and $\operatorname{domdim} A \geq n$ if there exists an exact sequence

$$
0 \rightarrow A \rightarrow X_{0} \rightarrow X_{1} \rightarrow \cdots \rightarrow X_{n-1},
$$

where $X_{i} \in \operatorname{add} X, i=0, \ldots, n-1$. This last claim follows from [13, 7.7]. In particular, there exists an idempotent $e$ such that $A e$ is a projective-injective faithful module which is a direct sum of pairwise non-isomorphic indecomposable modules. Under these conditions, $A e$ is called a projective-injective minimal faithful module. Furthermore, a minimal faithful projective-injective module $A e$ (if it exists) has a double centralizer property if and only if $\operatorname{dom} \operatorname{dim} A \geq 2$ (see for example [10, Theorem 2]). A module $M \in A$-mod is called a generator if ${ }_{A} A \in \operatorname{add}_{A} M$. Analogously, a module $M \in A$-mod is called a cogenerator if $D A \in \operatorname{add}_{A} M$. For self-injective algebras the notions of generator and cogenerator coincide.

Theorem 2 (Morita-Tachikawa correspondence) [10, Theorem 2] There is a bijection:

$$
\left\{\begin{array}{c}
\text { B finite dimensional } \\
k \text {-algebra } \\
M \text { a B-generator-cogenerator }
\end{array}\right\} / \sim_{1} \longleftrightarrow\left\{\begin{array}{c}
\text { A finite dimensional } \\
A: \begin{array}{l}
k \text {-algebra } \\
\text { domdim } A \geq 2
\end{array}
\end{array}\right\} / \sim_{2}
$$


Here, $A \sim_{2} A^{\prime}$ if and only if $A$ and $A^{\prime}$ are isomorphic, whereas, $(B, M) \sim_{1}\left(B^{\prime}, M^{\prime}\right)$ if and only if there is an equivalence of categories $F: B$-mod $\rightarrow B^{\prime}$-mod such that $M^{\prime}=F M$.

$$
\begin{aligned}
(B, M) & \mapsto A=\operatorname{End}_{B}(M)^{o p} \\
\left(\operatorname{End}_{A}(N), N\right) & \leftrightarrow A
\end{aligned}
$$

where $N$ is a minimal projective-injective faithful right A-module.

Usually, the Morita-Tachikawa correspondence is formulated for basic algebras. However, the above formulation is also equivalent due to a double centralizer property being a Morita invariant property.

Theorem 3 [13, 10.1] Let $A$ and $B$ be finite-dimensional k-algebras. Suppose that there is an equivalence $H: A$-mod $\rightarrow B$-mod. If there is a double centralizer property on $M \in$ $A$-mod then there is a double centralizer property on $H M \in B$-mod.

\section{Covers}

The theory of covers was introduced by Rouquier [11].

Lemma 4 [11, Proposition 4.33] Let $A$ and $B$ be finite-dimensional $k$-algebras such that $B=\operatorname{End}_{A}(P)^{o p}$, for some $P \in A$-proj. Denote by $F$ the Schur functor $\operatorname{Hom}_{A}(P,-): A$-mod $\rightarrow B$-mod and denote by $G$ its right adjoint $\operatorname{Hom}_{B}(F A,-)$. The following assertions are equivalent.

(i) The canonical map of algebras $A \rightarrow \operatorname{End}_{B}(F A)^{o p}$, given by $a \mapsto(f \mapsto f(-) a)$, $a \in A, f \in F A$, is an isomorphism of $k$-algebras.

(ii) For all $M \in A$-proj, the unit $\eta_{M}: M \rightarrow G F M$ is an isomorphism of A-modules.

(iii) The restriction of $F$ to $A$-proj is full and faithful.

Definition $5 \mathrm{We}$ say that $(A, P)$ is a cover of $B$ if the restriction of $F=\operatorname{Hom}_{A}(P,-): A$-mod $\rightarrow B$-mod to $A$-proj is full and faithful.

Remark 6 In the situation of Definition 5, a double centralizer property holds on $F A$, but not necessarily on $P$.

Before we proceed with some basic results about covers, recall the following result.

Proposition 7 Let $M, N \in A$-mod with $\operatorname{add}_{A} M=\operatorname{add}_{A} N$. Then the algebras $B:=$ $\operatorname{End}_{A}(M)^{o p}$ and $C:=\operatorname{End}_{A}(N)^{o p}$ are Morita equivalent and the algebras $\operatorname{End}_{B}(M)$ and $\operatorname{End}_{C}(N)$ are isomorphic.

Proof See for example [8, Proposition 1.3].

Proposition 8 Let $A$ be a $Q F-3$ algebra with a projective-injective faithful right A-module $V$. If $\operatorname{domdim} A \geq 2$ then $\left(A, \operatorname{Hom}_{A}(V, A)\right)$ is a cover of $B:=\operatorname{End}_{A}(V)$. 
Proof Let $e A$ be the minimal right projective-injective faithful $A$-module. Since $\operatorname{dom} \operatorname{dim} A \geq 2$ there is a double centralizer property $\operatorname{End}_{e A e}(e A)^{o p} \simeq A$. Because of $V$ being faithful projective-injective, add $V_{A}=\operatorname{add} e A_{A}$. By Proposition $7, \operatorname{End}_{e A e}(e A)^{o p} \simeq$ $\operatorname{End}_{B}(V)^{o p}$. Thus,

$$
A \simeq \operatorname{End}_{e A e}(e A)^{o p} \simeq \operatorname{End}_{B}(V)^{o p} \simeq \operatorname{End}_{B}\left(\operatorname{Hom}_{A}\left(\operatorname{Hom}_{A}(V, A), A\right)\right)^{o p} .
$$

The last isomorphism follows from $V$ being right $A$-projective and therefore $V$ being reflexive, that is, $V \simeq \operatorname{Hom}_{A}\left(\operatorname{Hom}_{A}(V, A), A\right)$. Further, this isomorphism is also an isomorphism of $B$-modules. So, the claim follows.

The definition of cover can be formulated in general for finitely generated projective algebras over commutative Noetherian rings. Unlike the general case, covers of finite-dimensional algebras can always be reduced to covers arising from idempotents.

Proposition 9 If $(A, P)$ is a cover of $B$ then there exists an idempotent $e \in A$ such that $(A, A e)$ is a cover of $e$ Ae and e Ae is Morita equivalent to $B$.

Proof We can decompose $P$ into a direct sum of projective indecomposables $P_{1} \oplus \cdots \oplus P_{n}$. By the Krull-Remak-Schmidt Theorem, there is a subset $I$ of $\{1, \ldots, n\}$ so that $Q:=\bigoplus_{i \in I} P_{i}$ is an $A$-summand of $A$, where the modules $P_{i}, i \in I$, are pairwise non-isomorphic and $\operatorname{add} Q=\operatorname{add} P$. Moreover, there exists an idempotent $e \in A$ such that $A e \simeq Q$. Hence, the algebras $B$ and $e A e$ are Morita equivalent. The functor $\operatorname{Hom}_{B}\left(\operatorname{Hom}_{A}(P, A e),-\right): B$-mod $\rightarrow e A e-\bmod$ is an equivalence of categories. On the other hand, the canonical map $\operatorname{Hom}_{A}(A e, A) \rightarrow \operatorname{Hom}_{B}(F(A e), F A)$ is bijective. Moreover, it is an $e A e$-isomorphism. Therefore,

$$
\begin{aligned}
A & \simeq \operatorname{End}_{B}\left(\operatorname{Hom}_{A}(P, A)\right)^{o p} \simeq \operatorname{End}_{e A e}\left(\operatorname{Hom}_{B}\left(\operatorname{Hom}_{A}(P, A e), \operatorname{Hom}_{A}(P, A)\right)\right)^{o p} \\
& =\operatorname{End}_{e A e}\left(\operatorname{Hom}_{B}(F(A e), F A)\right)^{o p} \simeq \operatorname{End}_{e A e}\left(\operatorname{Hom}_{A}(A e, A)\right)^{o p} .
\end{aligned}
$$

As mentioned, covers can be used to obtain properties of the module category of an algebra using one of its covers, for example, the number of blocks, or classification of simple modules, among many others. Although we do not pursue this direction here, cover theory really shines when the cover has finite global dimension and the algebra $B$ has not. For self-injective algebras $B$, covers of $B$ with finite global dimension are the non-commutative resolutions of [3]. As in their particular case, covers are non-commutative unless the cover of $B$ is isomorphic to $B$ itself.

Proposition 10 Suppose that $A$ is a finite-dimensional commutative $k$-algebra. If $(A, A e)$ is a cover of eAe, for some idempotent e in A, then $A$ is isomorphic to eAe.

Proof The commutativity of $A$ implies that $e$ is a central idempotent and $e A e$ is commutative. If $(A, A e)$ is a cover of $e A e$ then

$$
A \simeq \operatorname{End}_{e A e}(e A)=\operatorname{End}_{e A e}\left(e^{2} A\right)=\operatorname{End}_{e A e}(e A e) \simeq e A e .
$$




\section{Morita Algebras and Nakayama Functor}

Morita algebras were introduced by Kerner and Yamagata in [8]. A finite-dimensional $k$ algebra $A$ is called a Morita algebra if it can be written as the endomorphism ring of a generator-cogenerator over some self-injective algebra. A detailed account on Morita algebras and double centralizer properties can also be found in [15]. A characterization of dominant dimension over Morita algebras in terms of cohomology over self-injective algebras was given in [5].

For the proof of the main result, we require the following characterisation of Morita algebras. Theorem 11 is an extension of Proposition 2.9 of [4] (formulated in a different terminology).

Theorem 11 Let $A$ be a $Q F-3$-algebra. Let $P$ be a faithful projective-injective left $A$ module. The following assertions are equivalent.

(a) $\operatorname{domdim} A \geq 2$ and the Nakayama functor restricts to $D A \otimes_{A}-: \operatorname{add}_{A} P \rightarrow \operatorname{add}_{A} P$.

(b) $\operatorname{domdim} A \geq 2$ and $\operatorname{add}_{A} D A \otimes_{A} P=\operatorname{add}_{A} P$.

(c) The endomorphism algebra $B=\operatorname{End}_{A}(P)^{o p}$ is self-injective with generator $P \in$ $\bmod (B)$ and $A \simeq \operatorname{End}_{B}(P)$, that is, $A$ is a Morita algebra.

(a') The Nakayama functor restricts to $-\otimes_{A} D A: \operatorname{add} D P_{A} \rightarrow \operatorname{add} D P_{A}$ and $\operatorname{dom} \operatorname{dim} A \geq 2$. (b') $\operatorname{dom} \operatorname{dim} A \geq 2$ and $\operatorname{add} D P \otimes_{A} D A_{A}=\operatorname{add} D P_{A}$.

Proof We will show $(b) \Longrightarrow(a) \Longrightarrow(c) \Longrightarrow(b)$. The implications $\left(b^{\prime}\right) \Longrightarrow$ $\left(a^{\prime}\right) \Longrightarrow(c) \Longrightarrow\left(b^{\prime}\right)$ are analogous since $\operatorname{End}_{B}(P) \simeq \operatorname{End}_{B}(D P)^{o p}$ and cogenerators are exactly the generators for self-injective finite dimensional algebras.

The implication $(b) \Longrightarrow(a)$ is clear since $D A \otimes_{A} X \in \operatorname{add} D A \otimes_{A} P=\operatorname{add} P$, for all $X \in \operatorname{add}_{A} P$.

Assume that $(a)$ holds. Write $B=\operatorname{End}_{A}(P)^{o p}$. Let $A e$ be a minimal faithful projectiveinjective module. Then $\operatorname{add} A e=\operatorname{add} P$. By Proposition $7, \operatorname{End}_{B}(P) \simeq \operatorname{End}_{e A e}(A e)$. By Morita-Tachikawa correspondence,

$$
\operatorname{End}_{B}(P) \simeq \operatorname{End}_{e A e}(A e) \simeq A,
$$

and $A e$ is a generator of $e A e$. Since equivalence of categories preserves generators, $P$ is a generator of $B$. It remains to show that $B$ is self-injective. This follows by observing that, as right $B$-modules,

$$
B=\operatorname{Hom}_{A}(P, P) \simeq \operatorname{Hom}_{A}(P, A) \otimes_{A} P \simeq D\left(D A \otimes_{A} P\right) \otimes_{A} P \in \operatorname{add}\left(D P \otimes_{A} P\right)_{B},(6)
$$

where the third isomorphism is obtained by applying the functor $-\otimes_{A} P: \bmod -A \rightarrow \bmod -B$ and using Tensor-Hom adjunction. Moreover, by Tensor-Hom adjunction there exists a $(B, B)$-bimodule isomorphism $D\left(D P \otimes_{A} P\right)=\operatorname{Hom}_{k}\left(D P \otimes_{A} P, k\right) \simeq$ $\operatorname{Hom}_{A}(P, D D P)=B$. In particular, as right $B$-modules, $D B \simeq D D\left(D P \otimes_{A} P\right) \simeq$ $D P \otimes_{A} P$. So, $B \in \operatorname{add} D B_{B}$. Hence $B$ is $B$-injective.

Finally, assume that $(c)$ holds. Let $A e$ be a minimal faithful projective-injective module. Again, since $\operatorname{add}_{A} A e=\operatorname{add}_{A} P, e A e$ is Morita equivalent to $B$. So $A e$ is a generator of $e A e$ and $A \simeq \operatorname{End}_{B}(P) \simeq \operatorname{End}_{e A e}(A e)$. By Morita-Tachikawa correspondence, $\operatorname{domdim} A \geq 2$. Again, since $A \simeq \operatorname{End}_{B}(P)$ there exists an $(A, A)$-bimodule isomorphism $D A \simeq P \otimes_{B} D P$. 
Moreover, as left $A$-modules,

$$
D A \otimes_{A} P \simeq P \otimes_{B} D P \otimes_{A} P \simeq P \otimes_{B} D B .
$$

Since $D B$ is $B$-projective and $B \in \operatorname{add} D B, D B$ is a $B$-progenerator. Hence, $\operatorname{add}_{A} D A \otimes_{A} P=\operatorname{add}_{A} P$. This completes the proof.

Remark 12 By Tensor-Hom adjunction, for each $M, N \in A$-mod, the $\left(\operatorname{End}_{A}(N)^{o p}, \operatorname{End}_{A}(M)^{o p}\right)$-bimodules $\operatorname{Hom}_{k}\left(D M \otimes_{A} N, k\right)$ and $\operatorname{Hom}_{A}\left(N, \operatorname{Hom}_{k}(D M, k)\right)$ are isomorphic.

Using the terminology of [4], Theorem 11 says that all faithful projective-injective modules over a Morita algebra are strongly projective-injective. In particular, this provides a new and shorter proof for Proposition 2.9 of [4].

\section{Proof of the Main Theorem}

Proof of Theorem 1 The equivalence (ii) $\Leftrightarrow$ (iii) follows from the definition of Morita algebras and the Morita-Tachikawa correspondence. The equivalence $(i i) \Leftrightarrow(i v) \Leftrightarrow(v)$ is the content of Theorem 11 .

Assume that $A$ is a Morita algebra. By Theorem 11, add $D A \otimes_{A} P=\operatorname{add} P$. Let $A e$ be a minimal projective-injective faithful module. Then $\operatorname{addHom}_{A}(P, A)_{A}=\operatorname{add} D P_{A}=$ $\operatorname{add} D(A e)_{A}$. Since $\operatorname{dom} \operatorname{dim} A \geq 2$, we can write

$$
A \simeq \operatorname{End}_{e A e}(A e) \simeq \operatorname{End}_{e A e}(D(A e))^{o p} \simeq \operatorname{End}_{B}\left(\operatorname{Hom}_{A}(P, A)\right)^{o p} .
$$

This shows that $(A, P)$ is a cover of $B$.

Conversely, suppose that $(A, P)$ is a cover of $B:=\operatorname{End}_{A}(P)^{o p}$. By Lemma 4, there is a double centralizer property on $\operatorname{Hom}_{A}(P, A)$. More precisely,

$$
\operatorname{End}_{A}\left(\operatorname{Hom}_{A}(P, A)\right) \simeq B \quad \operatorname{End}_{B}\left(\operatorname{Hom}_{A}(P, A)\right)^{o p} \simeq A .
$$

In particular, $\operatorname{Hom}_{A}(P, A)$ is faithful-projective as right $A$-module. Hence, there exists an injective $A$-homomorphism $A \rightarrow \operatorname{Hom}_{A}(P, A)^{s}$, for some $s>0$. Since $D P$ is projective as right $A$-module, there is a monomorphism $D P \rightarrow A^{t} \rightarrow \operatorname{Hom}_{A}(P, A)^{s t} . D P$ is injective as right $A$-module. Hence, $D P \in \operatorname{addHom}_{A}(P, A)_{A}$.

We claim now that $D A \otimes_{A} P$ is a left $A$-projective module. To see this, define $P^{\prime}$ to be the direct sum of all non-isomorphic indecomposable $A$-modules that belong to the additive closure of $P$. So, add $P=\operatorname{add} P^{\prime}$ and $P^{\prime} \in \operatorname{add}_{A} D A \otimes_{A} P=\operatorname{add}_{A} D A \otimes_{A} P^{\prime}$. By KrullRemak-Schmidt theorem, we can write $D A \otimes_{A} P^{\prime} \simeq P^{\prime} \oplus X$, for some $A$-module $X$. On the other hand,

$$
\operatorname{End}_{A}\left(P^{\prime} \oplus X\right)^{o p} \simeq \operatorname{End}_{A}\left(D A \otimes_{A} P^{\prime}\right)^{o p} \simeq \operatorname{End}_{A}\left(\operatorname{Hom}_{A}\left(P^{\prime}, A\right)\right) \simeq \operatorname{End}_{A}\left(P^{\prime}\right)^{o p}
$$

So, by comparing $k$-dimensions, $X$ must be the zero module. Hence, $D A \otimes_{A} P^{\prime}$ is a faithful projective-injective module. Consequently, $D A \otimes_{A} P$ is also a faithful projective-injective module. Now, the double centralizer property (9) implies that $\operatorname{dom} \operatorname{dim} A \geq 2$. Since both $P$ and $D A \otimes_{A} P$ are faithful projective-injective modules, $\operatorname{add}_{A} P=\operatorname{add}_{A} D A \otimes_{A} P$. So, $A$ is a Morita algebra by Theorem 11 . 
Remark 13 For a idempotent $e$ of $A, \operatorname{Hom}_{A}(A e, A) \simeq e A$ as $(e A e, A)$-bimodules. In addition, assume that $e$ is an idempotent so that $A e$ or $e A$ is projective-injective. By Theorem 1, a double centralizer property on $A e$ is not equivalent to a double centralizer property on $e A$ unless $A$ is a Morita algebra. In particular, if $A$ is a Morita algebra then $A=\operatorname{End}_{e A e}(e A)^{o p}=\operatorname{End}_{e A e}(A e)$.

\section{An Application and Two Examples}

A finite-dimensional $k$-algebra is called $Q F-1$ algebra if all faithful $A$-modules have the double centralizer property (see [14]).

Corollary 14 Let $A$ be a $Q F-1$-algebra. Assume that $P$ is a faithful projective-injective left A-module. Then, $A$ is self-injective if and only if $\operatorname{Hom}_{A}(P, A)$ is a faithful right $A$ module.

Proof One direction is clear. Assume that $\operatorname{Hom}_{A}(P, A)$ is faithful. Since $A$ is a QF-1 algebra, $(A, P)$ is a cover of $\operatorname{End}_{A}\left(\operatorname{Hom}_{A}(P, A)\right) \simeq \operatorname{End}_{A}(P)^{o p}$. By Theorem 1, $A$ is a Morita algebra. By [1, Proposition 2.2], a Morita algebra is QF-1 if and only if it is self-injective. Therefore, $A$ is self-injective.

Example 15 For a QF-3 algebra $A$ with dominant dimension two and with a projectiveinjective faithful module $P$ the pair $(A, P)$ is not, in general, a cover of $\operatorname{End}_{A}(P)^{o p}$.

Let $k$ be an algebraically closed field. Let $A$ be the following bound quiver $k$-algebra

$$
1 \stackrel{\alpha_{1}}{\longrightarrow} 2 \stackrel{\alpha_{2}}{\longrightarrow} 3, \alpha_{2} \alpha_{1}=0
$$

Note that we read the arrows in a path like morphisms, that is, from right to left.

Denote by $P(i)$ the projective indecomposable module associated with the vertex $i$ and denote by $I(i)$ the indecomposable injective module associated with the vertex $i$.

The indecomposable projective (left) modules are given by

$$
\begin{gathered}
P(1)=I(2)=\frac{1}{2}, \quad P(2)=I(3)=\begin{array}{l}
2 \\
3
\end{array}, \quad P(3)=3 . \\
0 \rightarrow A \rightarrow P(1) \oplus P(2) \oplus P(2) \rightarrow P(1) \rightarrow I(1) \rightarrow 0
\end{gathered}
$$

is a minimal injective resolution of $A$. Denote by $P$ the projective module $P(1) \oplus P(2)$. Hence, $A$ is a QF-3 algebra with minimal faithful projective-injective left $A$-module $P$ and with $\operatorname{domdim} A \geq 2$. Here $B=\operatorname{End}_{A}(P)^{o p}$ is the path algebra with quiver

$$
1 \stackrel{\alpha_{1}}{\longrightarrow} 2 \text {. }
$$

But $B$ is not self-injective. By Theorem $1,(A, P)$ is not a cover of $B$. But, $(A, P(2) \oplus P(3))$ is a cover of $\operatorname{End}_{A}(P)^{o p}$ by Proposition 8. In fact, $P(2) \oplus P(3) \simeq \operatorname{Hom}_{A}(D A, P)=$ $\operatorname{Hom}_{A}(D P, A)$ as left $A$-modules.

This example also shows that $\operatorname{End}_{B}\left(\operatorname{Hom}_{A}(P, A)\right)^{o p}$ is not isomorphic to $\operatorname{End}_{B}(P)$, in general. 
Remark 16 If we drop the injectivity of $A e$ and of $e A$ in Remark 13, the statement is false. This can be seen in the next example.

Example 17 There are idempotents $e$ and non-Morita algebras $A$ so that there are double centralizer properties on $A e$ and on $e A$.

Let $k$ be an algebraically closed field. Let $A$ be the following bound quiver $k$-algebra

$$
1 \underset{\beta}{\stackrel{\alpha}{\rightleftarrows}} 2 \underset{\theta}{\stackrel{\gamma}{\rightleftarrows}} 3, \quad \gamma \alpha=\beta \theta=\alpha \beta=\gamma \theta=0 .
$$

We are using the same notation as in the previous example. So, the indecomposable projective (left) modules are given by

$$
P(1)=A e_{1}=\begin{aligned}
& 1 \\
& 2 \\
& 1
\end{aligned}, \quad P(2)=A e_{2}=\begin{aligned}
& 2 \\
& 3
\end{aligned} \quad \begin{aligned}
& 3 \\
& 2
\end{aligned} \quad P(3)=A e_{3}=\begin{aligned}
& 3 \\
& 2
\end{aligned} .
$$

The projective $P(3)$ has dominant dimension zero so $A$ cannot be a Morita algebra. We can see that $A$ has an involution fixing the primitive idempotents and interchanging $\alpha$ with $\beta$ and $\gamma$ with $\theta$. Fix $e=e_{1}+e_{2}$. By a direct computation, we can see that $(A, P(1) \oplus P(2))$ is a cover of $B=e A e$. Here, $B$ is the bound quiver $k$-algebra

$$
1 \underset{\beta}{\stackrel{\alpha}{\rightleftarrows}} 2 \longmapsto t, \quad \alpha \beta=\beta t=t \alpha=0 .
$$

Again by a direct computation or by observing that the duality of $A$ restricts to one of $B$ fixing $e$ it follows that $\operatorname{End}_{e A e}(A e) \simeq \operatorname{End}_{e A e}(e A)^{o p} \simeq A$.

Acknowledgments The results of this paper are contained in the author's forthcoming $\mathrm{PhD}$ thesis, financially supported by Studienstiftung des Deutschen Volkes. The author would like to thank Steffen Koenig for all his comments and suggestions towards improving this manuscript.

Funding Open Access funding enabled and organized by Projekt DEAL.

Data availability Data sharing not applicable to this article as no datasets were generated or analysed during the current study.

Open Access This article is licensed under a Creative Commons Attribution 4.0 International License, which permits use, sharing, adaptation, distribution and reproduction in any medium or format, as long as you give appropriate credit to the original author(s) and the source, provide a link to the Creative Commons licence, and indicate if changes were made. The images or other third party material in this article are included in the article's Creative Commons licence, unless indicated otherwise in a credit line to the material. If material is not included in the article's Creative Commons licence and your intended use is not permitted by statutory regulation or exceeds the permitted use, you will need to obtain permission directly from the copyright holder. To view a copy of this licence, visit http://creativecommons.org/licenses/by/4.0/.

\section{References}

1. Chan, A., Marczinzik, R.: On representation-finite gendo-symmetric biserial algebras. Algebr. Represent. Theory 22(1), 141-176 (2019)

2. Cruz, T.: Schur-Weyl duality over commutative rings. Commun. Algebra 47(4), 1619-1628 (2019)

3. Dao, H., Iyama, O., Takahashi, R., Vial, C.: Non-commutative resolutions and Grothendieck groups. J. Noncommut. Geom. 9(1), 21-34 (2015) 
4. Fang, M., Hu, W., Koenig, S.: On derived equivalences and homological dimensions. J. Reine Angew. Math. 770, 59-85 (2021)

5. Fang, M., Kerner, O., Yamagata, K.: Canonical bimodules and dominant dimension. Trans. Am. Math. Soc. 370(2), 847-872 (2018)

6. Green, J.A.: Polynomial Representations of $\mathrm{GL}_{n}$, volume 830 of Lecture Notes in Mathematics. Springer, Berlin (1980)

7. Koenig, S., Slungård, I.H., Xi, C.: Double centralizer properties, dominant dimension, and tilting modules. J. Algebra 240(1), 393-412 (2001)

8. Kerner, O., Yamagata, K.: Morita algebras. J. Algebra 382, 185-202 (2013)

9. Morita, K.: Duality for modules and its applications to the theory of rings with minimum condition. Sci. Rep. Tokyo Kyoiku Daigaku, Sect. A 6, 83-142 (1958)

10. Mueller, B.J.: The classification of algebras by dominant dimension. Can. J. Math. 20, 398-409 (1968)

11. Rouquier, R.: q-Schur algebras and complex reflection groups. Mosc. Math. J. 8(1), 119-158 (2008)

12. Soergel, W.: Kategorie $\mathcal{O}$, perverse Garben und Moduln über den Koinvarianten zur Weylgruppe. J. Am. Math. Soc. 3(2), 421-445 (1990)

13. Tachikawa, H.: Quasi-Frobenius rings and generalizations. QF-3 and QF-1 rings. Notes by Claus Michael Ringel, vol. 351. Springer, Cham (1973)

14. Thrall, R.M.: Some generalizations of quasi-Frobenius algebras. Trans. Am. Math. Soc. 64, 173-183 (1948)

15. Yamagata, K., Kerner, O.: Morita theory, revisited. In: Expository lectures on representation theory. Maurice Auslander distinguished lectures and international conference, Woods Hole Oceanographic Institute, Quisset Campus, Falmouth, MA, USA, April 25-30, 2012, pp. 85-96. American Mathematical Society (AMS), Providence (2014)

Publisher's Note Springer Nature remains neutral with regard to jurisdictional claims in published maps and institutional affiliations. 